\title{
Editorial
}

\section{Introducing Our New Chief Editor}

\author{
Josep M. Trigo-Rodríguez $\mathbb{D}$ \\ Institute of Space Sciences (IEEC-CSIC), Catalonia, Spain \\ Correspondence should be addressed to Josep M. Trigo-Rodríguez; aa@hindawi.com
}

Received 2 April 2020; Accepted 2 April 2020; Published 30 June 2020

Copyright (c) 2020 Josep M. Trigo-Rodríguez. This is an open access article distributed under the Creative Commons Attribution License, which permits unrestricted use, distribution, and reproduction in any medium, provided the original work is properly cited.

Advances in Astronomy is pleased to announce the appointment of Prof Josep Trigo Rodríguez as its new Chief Editor. In this Editorial, Prof Trigo introduces himself, describes some of the journal's journey and current status, and shares his vision and aspirations for its future.

I was delighted to be invited to become Chief Editor for the prestigious journal Advances in Astronomy and truly appreciate the opportunity to address this editorial to all of you. For those of you unfamiliar with my background, I'm an astrophysicist specializing in cosmochemistry and meteoritics and am currently working at the Institute of Space Sciences (IEEC-CSIC) in Barcelona, Catalonia. I have extensive experience in scientific publishing and am currently an Associate Editor for a number of journals within my research field. My research group studies the origin of planetary systems from evidence preserved in primitive meteorites, as well as the delivery of water and other volatiles to water-rich planetary environments, for example, Ceres and Mars. I hope that, in my new role, I can motivate colleagues and other researchers working in planetary sciences to submit their research to this journal. I'd like to emphasize here why I think that it is worthwhile doing so, particularly from my personal experience.

I have worked with Advances in Astronomy as an Academic Editor for about ten years and, behind the scenes, have been supported throughout by a team of professional and enthusiastic staff, helping with all aspects of the peerreview process. This care-which I have myself experienced as both an author and editor-is what enables a high level of service and ensures that we adhere to best publishing practices. One important aspect is Hindawi's effort to deliver the best reviewers and a strong emphasis on research integrity issues throughout the process. As a consequence of our editorial standards, only about one-third of submitted papers are accepted for publication, and on average, we're currently able to coordinate the full peer-review process within three months of submission.

Advances in Astronomy covers a broad scope and publishes high-quality research from right across astronomy, astrophysics, and cosmology. Since its conception in January 2008, the journal has grown to become a top open access destination for publishing original research and review articles. Throughout my tenure, I hope to drive $\mathrm{Ad}$ vances in Astronomy to better serve the astronomy, astrophysics, and planetary science communities. As well as pushing to deliver the latest technical services and Open Science initiatives, I will look to revise and refresh the journal's scope and direct it to follow the evolution of our discipline, for example, to encourage more papers on space missions, exoplanet research, and Solar System exploration.

There are many reasons to decide to submit to the journal. Given that the journal operates a fully open access publishing model, publishing in Advances in Astronomy provides maximum visibility, disseminating authors' research to the widest possible audience through its inclusion in numerous databases such as Web of Science and Scopus, without the need for a subscription. Finally, by publishing under Hindawi's default Creative Commons Attribution Licence (CC-BY), authors retain the copyright of their work, meaning they can grant anyone the right to reproduce and disseminate their research.

There have recently been several major achievements that, I hope, suggest a great future for the journal, and these include faster turnaround times in peer review and production, as well as improvements to our journal metrics 
including Impact Factor and Citescore. An increasing number of outstanding researchers have decided to publish their papers in Advances in Astronomy, which I firmly believe is a direct consequence of the right policies taken lately by the Editorial Board, and I'm now glad to participate in the progress of the journal from my newly appointed position. In that regard, the recent increases of the journal Impact Factor, which currently stands at over 2, alongside increases in other metrics including downloads and access to primary papers, consolidates the effort made by our editorial team and provides an additional argument to submit seminal papers to Advances in Astronomy.

In conclusion, I think that Advances in Astronomy provides an excellent destination for researchers across the breadth of the field to publish their research whilst benefiting from the rapid open access publication process. I sincerely hope that my incorporation as Chief Editor, and the subsequent changes to further improve the journal, motivates authors in the fields of planetary sciences and astrophysics to submit their research. From the quality of papers and Special Issues published recently, I think that Advances in Astronomy will positively contribute to the progress of observational and theoretical astronomy and space exploration. 